\title{
THE ACQUISITION, PRODUCTION AND DISSEMINATION OF GEOSPATIAL DATA FOR EMERGENCY MANAGEMENT AND PRESERVATION OF CULTURAL HERITAGE
}

\author{
E. Santoro \\ Geographical Information Department, Italian Military Geographical Institute, via C. Battisti 10, Firenze, Italy \\ enzo.santoro57@gmail.com
}

KEY WORDS: Geospatial data, Emergency management, Preservation of Cultural Heritage, Data-sharing

\begin{abstract}
:
The crisis management of a disaster, whether caused naturally or by human action, requires a thorough knowledge of the territory involved, with regard to both its terrain and its developed areas.

Therefore, it is essential that the National Mapping and Cadastral Agencies (NMCAs) and all other public and scientific institutions responsible for the production of geospatial information closely co-operate in making their data in that field available. This crucial sharing of geographic information is a top-level priority, not only in a disaster emergency situation, but also for effective urban and environmental planning and Cultural Heritage protection and preservation.

Geospatial data-sharing, responding to the needs of all institutions involved in disaster surveying operations, is fundamental, as a priority, to the task of avoiding loss of human lives. However, no less important is the acquisition, dissemination and use of this data, in addition to direct, "in-the-field" operations of specialists in geomatics, in order to preserve the Cultural Heritage located in the crisis area. It is in this context that an NMCA such as the Italian Military Geographic Institute (IGMI) plays a key role
\end{abstract}

\section{INTRODUCTION}

The painful experiences of repeated and "regular" catastrophies that make Italy a country of inherently high risk proposes in dramatic terms, especially in times of emergency, the ability to count on knowledge and on the availability of adequate and effective documentation and resources, taking into account the role the race against time plays with regard to those needs.

Calamities, sometimes announced, result in a greater sensitivity to the issue of risk prevention in an area; they, at the same time have raised awareness that not only natural phenomena - for example, storms, floods, landslides, volcanic eruptions, or earthquakes - cause disasters, but also the misuse of resources, unchecked by man, as for example, the abandonment of hill agriculture, irrational and prolonged deforestation, urban sprawl in areas of recognized geological instability or high flood risk - are, unfortunately, recurring realities (Surace, 2012a).

The catastrophic events of 2016 have confirmed, as if it were even necessary, that the activities to be carried out on the territory both during and after a calamitous event are less better than the work performed in "normal" times. What is necessary, therefore, is a different approach to crisis situations: terms such as "prevention", "local knowledge", and "ordinary operations" have to take the place of terms such as "exceptional phenomenon", "unforeseeable event", and "extraordinary intervention". In fact, one aspect to consider carefully is: all phenomena that are repeated at least every fifty years are to be considered "ordinary" and not "exceptional", (Surace, 2012b), contradicting the description, almost always, of a catastrophic event as "exceptional". In Italy, in recent years, this description has been applied to every flood and earthquake. It is the problem of "a short collective memory" that leads to forgetting what has happened some 20 years ago, perhaps because the visible effects (or wounds) caused by the event have been removed. It is a mental attitude that must be radically changed also because it is economically advantageous, simply taking into consideration the huge costs of the reconstruction of a disaster-prone area imposed upon the local community. However the economic issue is an aspect that goes beyond the arguments that will be dealt with in this article, that are the technical, procedural and organizational actions to be implemented in the area before, during and after a catastrophic event.

The management of crisis situations, whether due to natural disasters or human intervention, requires precise knowledge of the territory and its existing buildings. It is therefore necessary that all organizations/institutions which for varying reasons create, or manage, geospatial data, in any form or mode, work together in close coordination, so that these data are produced and distributed to aid "normal" management situations involving correct urban and environmental planning and the preparation of properly managed crisis situations. The need for shared geospatial data and the response to the needs of those institutions called upon to intervene on the territory of an emergency situation is a top priority to avoid loss of life.

No less important, however, are the acquistion, the dissemination and the use of geospatial data as well as the intervention in the field of specialists in geomatics for the preservation of historical and Cultural Heritage. Intervention in the field is important to realize appropriate metric surveying, to acquire high quality geospatial data designed to support civil protection operations, that, concerning the protection of Cultural Heritage in crisis areas, discussed further on, will consist of operations aimed at the safeguarding of the artifacts, and, in the worst cases, precise operations for their restoration or complete reconstruction. In such a scenario, up to the present, at the request of the Authorities, or in the performance of its institutional activities, the role of the Istituto Geografico Militare has been realized by "post-event" operations regarding specific and accurate activities of monitoring, restoring and remeasurement of geodetic networks and high-precision levelling of the areas concerned. These activities are aimed at creating correct territorial geometric frameworks after the 
catastrophic event, indispensable for any subsequent actions in crisis areas. These activities derive from the National Mapping Agency duties established by law of the $20^{\text {th }}$ February 1960 n. 68. This law, still in effect, assigned precise tasks and differing roles to the various National Mapping and Cadastral Agencies, that collected and produced their own (and often not shared) geospatial data, and varying type and scale maps whose contents remained largely confined only to the specific cartographic need. At the time there was no discussion of "interoperability", data "use and re-use", "derivability", "Data Bases", "information layers", data "standardization" and the INSPIRE Directive was yet to come. All of this certainly requires an observation on the limits and novelty that the law of 1960 demonstrates at present, which impose, in my view, a new redistribution of the skills and structure of the National Mapping Agencies and the Entities institutionally responsible both for the production of large-scale topographic and cartographic databases and for a new vision of Italian geographic information aimed at territorial knowledge and representation as well as meeting the demands for cartographic instruments focused on civil protection requirements and competent management of emergency situations resulting from catastrophical events.

With regard to this situation, a notable assistance could come from the "National Council for Territorial and Environmental Information", established under Article 11, paragraph 5, of Legislative Decree of $27^{\text {th }}$ January 2010, n. 32, the operational activities of which were established by Decree of the President of the Council of Ministers on the $12^{\text {th }}$ of January, 2016. As of the present, unfortunately, it has yet to meet and to carry out the tasks which were assigned.

Another point of discussion could be, at the national level, the governmental Act n. 30, of the $16^{\text {th }}$ of March, 2017 by which the government is authorized to issue specific decrees of reorganization of the national system of civil protection. In particular, it can refer to articles within the law regarding:

- the contributions of the University (Article 1, paragraph e):

"regulation of the participation and collaboration of Universities and entities and research institutions for civil protection activities, for the purpose of integration of knowledge and products resulting from research activities and innovation, also the result of initiatives promoted by the European Union and international institutions also in the field of research for the defense against natural disaster",

- measures for the re-establishment of the areas affected by disastrous events (Art. 1, paragraph m):

"regulation of the measures to be adopted in order to remove the obstacles for the restoration of the ordinary life conditions in areas affected by calamitous events. These measures include structural and non-structural interventions aimed at prevention and restoration activities, within the territories, of the damaged public works and infrastructures as well as of the ones of public interest, including the ones that can be considered strategic. Not to mention the reduction of the residual risk and the speed-up of the exiting from the emergency status.....omitted....";

- measures involving citizens (Art. 1, paragraph d):

"regulation of the participation and responsibilities of citizens, individually and associated, including associations of a professional nature, to civil protection activities, with reference to the planning of initiatives to be taken to deal with an emergency, to the practical activities, the dissemination of knowledge and the culture of civil protection in order to promote the resilience of communities, also with the awareness of the rights and duties and the adoption of self-protection measures, ... omitted ...".

This last paragraph is particularly important because it officially expresses the concept of "resilience" of a community.

These actions cannot realize concrete implementations without the availability of adequate, homogeneous and updated geospatial information regarding the National territory and its cartographic support at different scales.

\section{GEOSPATIAL DATA IN EMERGENCY MANAGEMENT}

2.1 What is needed in an emergency situation?

A by no means exhaustive list should include:

- medium and small scale cartography in digital format enabling the identification of the natural disaster area and the realization of early intervention plans;

- large scale updated cartography in vector format enabling data analysis and querying;

- high resolution $(50 \mathrm{~cm}$ or better) ortophotos;

- specific and uniform data layers of the affected area with special attention to the layer concerning viability and buildings; no less important, however, are the hydrography, land use and productive activities layers;

- cadastral cartography of the inherent characteristics covering the entire national territory and essential indications of building types, with primary focus on private dwellings and public edifices;

- statistical information regarding population, production, administrative boundaries, and census section data;

- digital terrain models (DTM) and digital surface models (DSM) of high to highest quality/accuracy for the correct realization of orthophotos of the affected area, and for detailed studies relating to slope stability analysis, useful for updating and verification of hydrological plans, for the design of work regarding soil protection and for the activities of land use planning at every level;

- during the event, the availability of every kind of open data (including, for example, Open Street Maps (OSM) and data created by the so-called VGI -Volunteer Geospatial Information);

- quickly available cartography, during, or post-event, regarding specific territorial situations to be realized on the spot, according to the specific local conditions/needs;

- use of surveys carried out by means of UAVs (Unmanned Aerial Vehicles);

- availability of authoritative (if possible) geospatial data produced in standard format for integration with other data.

What is needed with specific regard to Cultural Heritage, in addition to the requirements listed above?

- an updated database of all Italian Cultural Heritage (archaeological, architectural, landscape, historical and artistic, scientific and technological, ....) to be integrated with the data already present in the "Catalogue of Cultural Heritage" of the ICCD (Istituto Centrale per il Catalogo e la Documentazione) of the Ministry of Environmental and Cultural Heritage and Tourism (MiBACT) including at least georeferencing and surveying data, as well as, when possible, the creation of 3-D models;

- the creation of a "geomatic task force" which can operate in the field during the event, supporting facilities for emergency management (12 December 2013 MiBACT 
directive: "Procedures for managing safety and safeguarding of Cultural Heritage in emergencies arising from natural disasters" (GU n.75- March, 31 $\left.{ }^{\text {st }}, 2014\right)$;

- an improved culture of information-sharing with regard to activities, studies and surveys involving Cultural Heritage is needed which can be of assistance during the phases of both emergency management and subsequent reconstrution. Too often, in fact, duplicated surveys, involving the same object, are discovered due to the lack of data resulting from its loss or the its unclear customer-executor ownership.

\subsection{What is, presently, the situation regarding the avaibility of cartography and geospatial data in Italy ?}

- Medium-small scale cartography is available, as it is a specific task of the Italian Military Geographic Institute (IGMI); it is 1:25000 to 1:250000 scale mapping, often not sufficiently updated;

- large/very large scale mapping also exists: it is produced by Entities at regional and munipal levels of uneven quality and not sufficiently updated . It should be noted that the regional cartographic base $5 / 10 \mathrm{k}$ is the essential reference for operational and management purposes, not only for emergency aid, but also for policing activities and interventions in situations such as the presence of dangerous materials;

- presently is well-established the availability of high resolution $(50 \mathrm{~cm}$ or better) ortophotos produced by AGEA ( Agenzia per le Erogazioni in Agricoltura);

- specific information layers, if they exist, are often incomplete and/or uneven in quality, and, most certainly, do not cover the entire national territory;

- the cadastral maps and the information residing in cadastral databases covering the entire national territory and its use has been proven vital in emergencies, but is lacking the elevation component and, partially, in the toponymy (geographical names), greatly reduced in the transition from analogical products to digital ones. Also they lack the evidence of some buildings which are a consequence of urbanization abuse in spite of the action carried out in recent years by the Italian Cadastre that has led to millions of "ghost" buildings;

- statistical information, based on census documentation, is well-integrated with cadastral information and large scale cartography but it would need authoritative and well georeferenced data.

- the existing DTM, covering our entire national territory, is that with a Ground Sampling Distance (GSD) of 20 meters produced by IMGI during the 90 's; it has been derived thanks to a simple interpolation of the already existing contour lines and the elevation points. The high accuracy DTM (GSD of 1-2 meters) carried out by Light Detection And Ranging (LIDAR) methodology, produced by the Ministry of the Environment and Protection of Land and Sea (Ministero dell'Ambiente e della Tutela del Territorio e del Mare -MATTM) within the Remote Sensing Extraordinary Data Collection Plan (PST), presently covers only $46 \%$ of our national territory (Conte et alii, 2016a).

\section{MEETING EMERGENCY MAPPING REQUIREMENTS}

After a catastrophic event, the first step to accomplish is to activate a platform which allows the preparation of basic essential information, of both large scale and medium scale, beginning with the first rough estimate and selection of data, regarding, for example, the condition of roads and affected buildings.

In this first phase, it is necessary to prudently separate geographical information coming from a volunteer source from information deriving from an authoritative source.

It is essential to keep in mind that, for the Civil Protection Agency, a priority of information regarding the positioning of operational headquarters of human resources, logistics and instruments is necessary for the management of an emergency. Of particular importance is information regarding the main urban centers not directly affected by the event.

In addition, and before planning any intervention, it is vital to gather information regarding resident population, occupied buildings, strategic structures, travel distances, viability of road networks and their infrastructures, and pertinent geomorphologic aspects.

An information of fundamental importance is that of the road network; it is essential to realize a recognition and monitoring of the state of the road system and alternative travel routes, following precisely established protocols. Consequently there is a need to keep the conditions of road use constantly updated. In order to be prepared for an event of this type an authoritative graph of national coverage (as of the moment not completely available) needs to be available, in anticipation, containing all the information necessary to address a crisis situation, thus overcoming the management problems created by various data producers from multiple institutional levels. In particular, this national-level graph should be structured in vectorial format, combined with functional information concerning road network(s), toponomy and conditions and the state of preservation of travel routes. Moreover, it should not be too simplified, especially with regard to minor travel routes, in order to guarantee better planning for emergency situations, hopefully even listing street house numbers.

As of the present there are inadequacies regarding the Roads Cadastre instituted by the new Road Code (D. Lgs. 285/1992) to be structured following the Geographic Data File GDF standard as defined by the Ministry of Public Works Decree of June the $1^{\text {st }} 2001$.

Often in an emergency situation we resort to the use of the OSM (Open Street Map) which includes both primary and secondary roads. Unfortunately this instrument suffers from a lack of sufficiently trustworthy data.

With regard to built structures it is necessary to associate assessed buildings with their precise location (not only at the section census level), including street house numbers, information from cadastral mapping, following the same indentification. Up to the present, and, although much better than in the past, this situation unfortunately has not been entirely resolved. In point of fact, while the House Numbers Index containing the road graph, is not yet available, the DPCM of 12 May 2016 has established a National Archive of Street House Numbers and Urban Roads with addresses and uniform codificaton with other databases (Carlucci et alii, 2016).

As was previously stated, an overlapping of orthoimages with regional data deriving from national-level cadastral mapping is most assuredly useful in permitting the localization, property identification, and the status of local conditions well before a calamitous event.

\subsection{The use of large scale maps}

Large-scale mapping and topographic DBs on scale 1: 10000 are the basic references for all operational purposes during the emergency phase. 
As is well known, technical maps, unlike medium and small scale ones, represent elements in projection and not by a symbolic representation and therefore are the most suitable for all surveying activities and for implementing targeted intervention plans as well as for all planning activities needed for the following reconstruction phase.

The problem is that in most cases Regional Technical Maps (CTRs) are not up-to-date and are mostly not homogeneous between neighboring Regions.

This circumstance implies that in the emergency phase it is necessary to integrate such a kind of maps with other geospatial information and to immediately work by means of survey in the field, which is often not easy to do during the calamitous event.

\subsection{The use of Cadastral and ISTAT data}

As stated previously, after defining the environment of the emergency, the use of a specific cadastral map of the affected area comes into play.

Priority of use is given to cadastral mapping because it represents large scale authoritathive cartography, covering the nation's entire territory, and is available using interoperable services. (Furthemore, with its metadata it is also listed, using, national rules, in RNDT -Repertorio Nazionale dei Dati Territoriali- which will be discussed later).

The Italian Cadastre, as National Mapping Agency since 1960 , is responsible for cadastral mapping and its continuing updating.

The entire homogeneous cadastral mapping reference system, with its national coverage, is based on large-scale cartography involving over 300,000 map sheets at 1:2000 scale while urban maps are at 1:1000 scale. It is structured on a vector format system and is available for all local and global coordinate systems. It is linked to census and administrative archives by means of a unique national-level identification code, the cadastral parcel (Ferrante, Ambrosanio, 2016a)

It is important to emphasise that maps and related census archives are linked and must be updated continually and congruently.

At the present, cartographic and census archives are updated simultaneously by means of a procedure entitled PREGEO. Using PREGEO guarantees consistance in the flow of updated data. In the past, however, it must be admitted that there have been cases of misalignment between the mapping and census data of Land Cadastre (Catasto terreni), and the census data of the Urban Cadastre ( Catasto urbano)

The strategic importance of the use cadastral mapping has been by now well-established as was verified in the field during the earthquake of 2016 in central Italy. Coming from this last tragic event the National Revenue Agency and the Civil Defence Agency have stipulated a ten-year agreement establishing a regular and continuing relationship, whose purpose is to give value to all cadastral data, in particular mapping and technical data regarding buildings, in order to integrate cadastral data and fiscal data into the Civil Protection Department's information system, as well as to support that Department's intervention in an emergency event, for better use of cadastral data during the planning, emergency and post-emergency phases.

This agreement between the two agencies has found a practical application with the immediate use of cadastral mapping in an activity already underway: the use of Copernicus Emergency Management Service.
At the request of the Civil Protection Department the cadastral cartographic data for buildings has been used and reworked in the framework of the European Copernicus program in support of damage detection on the affected edifices and its related mapping-based thematization.

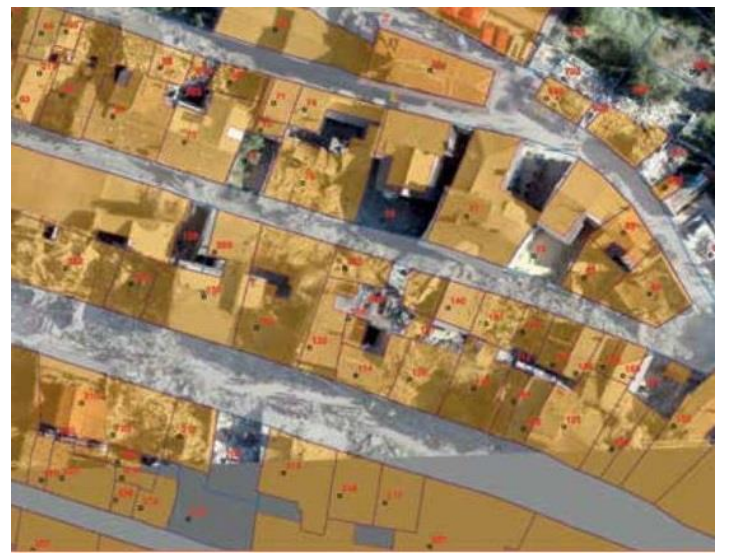

Figure 1. Example of cadastral data overlapping satellite image

In fact, cadastral map information, allowing the unique identification of any property (terrain or built), covering national-level territory, constitutes an indispensible element for the management of multiple technical and administrative procedures initiated in the phases following the arrival of first aid, and that must continue during the preparatory phase of reconstruction, beginning with the realization of reception areas for the installation of emergency infrastructure, the verification of building code compliance, the estimates of occupational benefits and the expropriation of occupied terrain.

Of particular interest is the bringing together of ISTAT statistical data (cadastral parcels, resident population, as well as the distribution of productive activities of the affected area.) In fact, the ISTAT mapping layer (entitled Territorial Base - BT) forms the official mapping support for data collection and dissemination operations recorded during a census. In this respect, BT is the representation of geographical objects that describe the settlement "mosaic" characterizing the country and its evolution in the medium/long term. Thus the BT is a valid instrument for realizing territorial planning for all that concerns the resident population, and for defining emergency planning. During the emergency event it is important to give priority to rescue activities in densely populated areas; in the post-event phase the BTs, if associated with other information sources can be of considerable support in the identification of areas suitable for the creation of temporary settlements or the location of housing modules.

Of notable interest are the platforms for sharing online cadastral mapping, accessible via the internet, to citizens, pertinent professionals, and public administrations. For the last two groups listed in particular, using the National Revenue Agency's portal, the "Interchange System" is available by means of cooperative applications services. Downloading services, free of charge for all Public Administrations, are related to vector cadastral mapping as well as census data of the Land Cadastre and the Urban Cadastre and metric data deriving from the Urban Real Estate Units (UIU) (Ferrante, Ambrosanio, 2016a).

Additionally, for all municipalities (excepting the autonomous provinces of Trento and Bolzano) a specific platform for free data exchange (the "Portale per i Comuni") 
makes it possible to download at no cost cadastral maps in vector format, Land Cadastre and Urban Land Registry census data, UIU metric data, updated records, transcript notes, and buildings never declared in the Land Registry for the territory involved: an optimal example of data sharing.

\subsection{The use of Voluntary Geographic/Geospatial Information (VGI)}

In the managment of emergency situations the use of VGI should not be underestimated, having as its benefits an extremely rapid acquisition and distribution of this information, which, in turn, is very useful for the prompt production of maps to assist in planning actions in limited areas.

Not to be overlooked, however, is the lack of data certification and scarce quality often found in VGI. The doubtful data quality in VGIs is often due to the difficulty of its certification. In point of fact, the protocols used in the production of geospatial data for official data sets are not respected in the VGIs. VGI data is collected by less rigorous means than those used to create official data sets; moreover, the data collectors/producers are rarely experts in geomatics. VGI data is, therefore, extremely heterogeneous regarding coverage and accuracy.

For these reasons, it is necessary to pay close attention to the correct use of this data, and, above all, to assure its integration with data originating from pre-existing official information and cartographic sources.

Among the VGIs the OpenstreetMap (OSM) should be noted as the most popular and widely-used VGI project. Initially OSM was focused solely on road mapping; presently it has been extended to any type of object which can be georeferenced.

The prolonged time-period of the 2016 earthquake in central Italy has meant that a notable number of volunteers have been involved in the production of VGIs, thus making an effective contribution to the operations of damage monitoring. These data instruments, integrated with geospatial information originating from official sources, were later to be used in the production of emergency mapping, within the Copernicus EMS project, to cover the earthquake impacted areas.

\subsection{The use of Drones}

The use of Unmanned Aerial Vehicle (UAV) together with the availability of Software for a rapid restitution and realization of 3D models ( like nowadays can be a Structure From Motion SW), is, by now, an established technique, speedy and extremely efficient in the realization of geospatial data indispensible in rescue operations typical of the first intervention phase after a calamitous event. UAVs are able to collect data in areas with difficult access; for example, in the risk-free evaluation of damage to buildings.

At this stage of intervention the rapidity of data acquisition leaves in the background the accuracy of precise metric survey.

At this intervention stage the rapidity of data acquisition takes priority, leaving for a later moment the accuracy of precise metric surveying.

It is necessary to keep clearly in mind this concept because, from time to time, the data processed can give apparently reliable results, hiding, however, geometric errors and deformations as in the case of images improperly acquired. Once the first-aid phase has ended drones can be used to acquire geospatial data resulting in their integration with pre- existing mapping or in process mapping and dedicated information systems.

In point of fact, the adoption of proper modalities of the use of such instrumentation, added to the rapid evolution of photogrammetric technologies related to the UAVs, permits users to obtain extremely complete and updated information in real time.

In this specific area it should be noted that the cartographic use of drones is desirable, both for the predisposition of operational bases on limited territorial areas involved in emergency, and for their rapid realization.

Not to be underestimated is the surveying use performed by UAVs, in addition to classical surveying methods, towards the safeguarding of architectural heritage affected by a disastrous event.

One should reflect upon the possibility to have a team of experts in geomatics, in the field, logically autonomous, working safely side by side with the Civil Protection operators, with the aim of carrying out metric surveying towards both the verification of the state of the impacted structures and their recreation in three-dimensional models.

\section{THE COPERNICUS EMS (EMERGENCY MANAGEMENT SERVICE) PROJECT}

Copernicus, previously known as GMES (Global Monitoring for Environment and Security), is a program coordinated and managed by the European Commission to provide Europe with the proper skills regarding the observation of the earth. This program, together with the satellite missions of SENTINEL (of which the Centro Spaziale di Matera is one of the three bases of the Core Ground Segment) includes operational services dedicated to the dissemination of products useful for professionals involved in the most important phases of ordinary and extraordinary territorial management. Copernicus Emergency Mapping, in operation since 2008, came into being following five years of experimentation.

This service provides the European Commission with geospatial information and satellite maps of all the areas struck by emergency, thus making available to the Civil Protection Agencies, pertinent local authorities of all Countries in the European Union and international humanitarian organizations the data necessary to manage a catastrophic event. The service, operational 24 hours a day, 365 days a year, having a productive capacity to deal with all types of crisis situations (floods, earthquakes, fires, technology disasters), is managed on behalf of the European Commisssion by the Italian company e-GEOS, at the head of a consortium consisting of the German society, GAF, the Italian society, Ithaca, and the French member, SIRS ( Cardillo et alii, 2016) .

The Copernicus Emergency Mapping Service, begun by the European Commission following a request from one of the European Union countries, provides digital maps (also ready for printing) and vector files referencing the situation preand post-event. The typology of the certified products available vary according to the the type of calamitous event which has occurred and can rapidly provide the delivery of:

- Reference maps, providing basic understanding of the territory and its patrimony based on data created predisaster such as linear, areal or punctual elements such as transportation networks, urban settlements and infrastructures;

- Delineation maps, providing a first estimation of the geographical extent of the area hit, on the basis of satellite images acquired soon after the disaster. The delineation 
maps indicate for example, flooded, fire-damaged or earthquake areas.

- Grading maps, providing an estimation of the impact and the order of magnitude of the damages caused by the event. The maps are derived from pre- and post-event satellite surveys. Thus the resulting product includes an evaluation of the measure, type and importance of specific damage(s) based upon the type of the calamitous event.

The Grading map shown in the Figure 2. is an example realized through a high-resolution aerial image acquired before the event compared with the higher resolution image acquired immediately after the calamitous event. The map portion shows the degree of damage to the area of the city of Preci with a visual interpretation made by some elements (colored polygons based on the degree of damage). Estimated final geometric accuracy is 1 meter or better, derived from the native resolution of aerial and satellite imagery used.

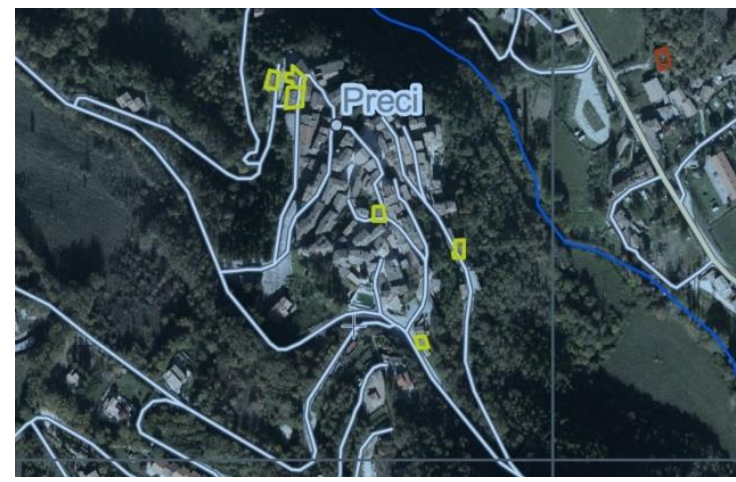

Figure 2. Grading map of the city of Preci

The Grading Map has been created using the following geodata:

- Pre-event image: Orthophotos with resolution of $50 \mathrm{~cm}$ carried out in 2014 by CONSORZIO TeA for AGEA.

- Post-event Image: Aerial Image of the European Commission provided under the COPERNICUS project by CGR, Compagnia Generale Riprese aeree S.P.A ..

- Vector Basic Layer: OpenStreetMap and GeoNames.

- Other datasets: EuroGeographics, Natural Earth 2012, CCM River DB EUJRC2007, Digital Elevation Model SRTM 30 m (NASA / USGS).

In addition, the Copernicus constellation Sentinel-1 twin radar satellites, combined with ground segment cloud computing, are able to monitor seismic areas for highprecision terrain detection.

Each time a movement is identified, more detailed controls are initiated, which can be done through the ESA's Geohazards Exploitation Platform.The satellites capture successive radar images of the same position that are then combined to reveal the minimum displacement.

There are three categories of users of the Copernicus EMS:

- Authorized Users: They can activate the service by contacting directly the Emergency Response Coordination Center (ERCC), operated by the DG ECHO, the European Direction for Humanitarian Help and Civil Protection.

- Associated Users: They can activate the service through an Authorized User, as public, state or regional agencies.

- General Public Users: They are not authorized to activate the service. However they have access to all information and data on current activities passed through the web portal: emergency.copernicus.eu.

With particular reference to the 2016 earthquake in central Italy the Copernicus EMS was immediately activated by the
Italian Civil Protection and by the Italian Space Agency, thus permitting the emergency center, after very few hours, to begin the territorial analysis activities using optical and radar satellite-acquired images resulting in an almost immediate response to the request.

The Civil Protection Agency took advantage to the fullest extent of the possibilities offered by the Copernicus EMS for the urgent provision of maps and vector cartography for use in the field. During the event the international team produced within the project more thn 100 maps of the impacted areas profiting fully from all available data on social media in order to obtain the most reliable information in the shortest possible time.

To be emphasized, in conclusion, is the important role played by the Italian COSMO-SkyMed satellites constellation, composed of four satellites equipped with Synthetic Aperture Radar operating at X-band,whose data was used to quickly evaluate the damage extent and impact, performing highprecision measuring, and assuming the responsibility for change detection (monitoring variations) between the two seismic events which followed.

\section{DISSEMINATION OF GEOSPATIAL DATA}

As is well-known, in Italy, geospatial information suffers from a fragmentation that is far from being resolved. Fragmentation of this data has been the subject of discussion among all data producers, and which is part of a need for a solution recognized at the European level. In point of fact, the European Commission, by Directive 2007/2/CE, better known as the INSPIRE Directive, has promoted in the last 10 years, the realization of territorial data infrastructures of the individual member States, viewed as the nodes of a unitary European infrastructure. The declared aims of the Directive are to facilitate access to geographic/geospatial information, making it easy to trace and quickly available.

The need for an adequate territorial data infrastructure is mainly felt in crisis management and emergency situations during which high-quality information - having also a geographic dimension - must be speedily acquired to support the decision-making process. In most cases, it is necessary to ensure that the data involved can be easily shared, especially if it derives from a variety of sources and relates to different territories. The emblematic case is the 2016 earthquake in central Italy involving four regions (Abruzzo, Lazio, Marche, and Umbria) with data imput both from their regional organizations and from central and local authorities having specific authorizations in the impacted areas.

Thus the crisis management team had to deal with the problem of harmonization and the use of geospatial data and cartographic productions having differing quality and lacking adequate updates.

All this despite the commitment in Italy in recent years to the adoption of shared technical standards to be used in training and harmonization of quality regional geospatial data.

Ensuring quality is not enough, however. It is also necessary to ensure what in English is called "discoverability" of data aiming at ease of access and use. In other words, in addition to the existance and high-quality of the data, assurance must be made that it is searchable and findable in order to evaluate its use to satisfy specific requirements.

The metadata catalog entitled the Repertorio Nazionale dei Dati Territoriali (RNDT), managed by the Agenzia per l'Italia Digitale (AGID) responds precisely to this central role (Ciasullo, Rotundo, 2016). This function becomes even more 
important when emergency events are involved.

A centralized point for accessing the available information, how and where to acquire it, reduces the time and effort spent in the search, which, as indicated in the preamble to the Directive INSPIRE, is a decisive obstacle to optimal data exploitation. In other words, if a national-level catalog did not exist, it would be necessary to seek information through the various (geo)portals (if available) of the individual administrations, similar to what was done in the past, and, in some cases, is still done in some public offices. As is known, such data is not directly accessible and usable in the sense that metadata generally provides a representation referring to availability. The possiblility to access and use the data is in accordance with the policy for transfer of the data to individual administrations.

The potentials of RNDT are not put to maximum usefulness because not all administrations allow complete access to their data. Thus further efforts are needed to ensure these conditions can be summarized in the following points:

- Continue to populate the RNDT with those administrations which do not yet permit full access to data and information. - Ensure the constant updating of metadata information.

- Ensure appropriate levels of data and metadata quality.

- Beyond simply representing the availability information held, adoption of access, sharing and re-use policy in line with the latest national and European-level legislation on public sector information (PSI)

The points above concern the awareness of the availability of geospatial data. Is there, in Italy, a national-level entity, in addition to producers, tasked with the distribution of territory data as stipulated in the Directive 2007/2EC of the European Community?

In Italy, the INSPIRE Directive has been adopted by legislative Decree conferring to Ministry of the Environment and Land and Sea Protection Protection (Ministero dell'Ambiente e della Tutela del Territorio e del Mare (MATTM) the role of competent authority for its implementation. Within the same Decree - in the cartographic portal of the same Ministry - the "Geoportale Nazionale" (GN) is identified as a national-level access point for territorial and environmental information (for the purposes of the Decree itself). From an institutional point of view, the Geoportale Nazionale represents the instrument for access to the geospatial data produced by public administrations. The GN, through the implementation of appropriate network services is able to share data, avoiding duplication, while preserving its contents and responsibilities to the supplying public administrations.

The process assumes that each data producer adapts its own database(s), each with its own metadata following defined metadata standards, with subsequent publication through network services based on standard OGC (Open Geospatial Consortium).

It is evident that the data in the GN are all validated by the producing organizations, and therefore "certified" as they derive from authoritative sources. Conversely they have a proper update time established by the supplying administration.

In this regard it is important to point out the predominantly economic nature of some geospatial data producers to adapt to standards and and to implement an infrastructure for their release. This is a paradoxical factor that hardly affects the large-scale cartographic producer because those maps are the most detailed and therefore the most useful in emergency situations.

The adoption of standards has been mentioned, and, in point of fact, standardization is the necessary condition for relating information of differing nature and derving from different sources. From this assertion come the objective that must be achieved by the Geoportale Nazionale del MATT, which is to represent a centralized national-level database, as complete as possible, capable of aggregating data from different public entities therough the sharing of rules and adoption in common of standards.

The GN database is composed of all the information collected over time in the context of several projects carried out by the MATTM (for example the Piano Straodinario di Telerilevamento Ambientale) and the contributions from over 300 negotiation protocols stipulated with central and local administrations for data exchange.

To date, the GN is composed of its own metadata catalog which offers the ability to seach for date of interest, of a WebGIS, which allows the visualization and retrieval of data to unknown users, and, finally, the the OGC standard network services that allow a more experienced audience (specialist users) to access information, sometimes with download capability, and to integrate them with other layers all by using a GIS client, including open source, such as the widely known QGIS (Conte et alii, 2016b). The availability of the PST database is verifiable through the GN and can be requested by simplely sending an e-mail.

It is clear that the committment of all geospatial data producers in Italy is to directly or indirectly promote the growth of the centralized GN database, and to make it ever more accessible to the so-named "non-experts" with the goal of a realistic policy of sharing both spatial information and best practices to be implemented in the ultimate result of making Italy more efficient in the planning, monitoring and emergency response phases.

\section{THE ROLE OF THE ITALIAN MILITARY GEOGRAPHIC INSTITUTE}

With reference to the above affirmations, some areas of activity undertaken by the Italian Militiary Geographic Institute (IGMI) to make a useful contribution both in emergency situations and in the prevention and prediction of natural disasters.

As previously mentioned, the role of the IGMI until now has come into being based on the request of the authorities and its institutional activities in post-event actions: providing small and medium scale maps, technical specifications and specific activities regarding the monitoring, restoration and leveling networks of the affected territory. These actions, aimed at the correct geometric framing of the impacted area following the catastrophic event are, moreover, indispensible for subsequent post-event operations.

The relatively limited role described above was probably due to the fact that the IGMI was viewed primarily as a cartographic agency in support of the Armed Forces, or perhaps as a result of the length of time needed for the creation/updating of the cartographic products (the quality and aesthetic aspects of the maps at 1:25000, the famous "tavolette", and those at 1:50000 came to be acknowledged as the most beautiful in Europe) was so long, taking decades for the completion of a mapping series.

The new scenarios involving the field of geomatics, the advancement of computer technology and the new official technical rules regarding the production of geospatial data to manage emergency situations subsequent to a calamitous event have led to a new role for the IGMI, particularly in the production of geospatial data.

To respond to each situation and to achieve desired 
objectives before, during and after the calamitous event the IGMI's own activities and its cartographic production are now the starting point, representing the common repository of geographic information made available to the various institutions involved in crisis management. The experience gained in the acquisition, simplification and post-processing of geospatial data - both in civil and in military fields - has made the IGMI an expert in the preparation of digital data organized in topographical databases, digital terrain models and cartographic products at various scales, all guaranteeing certified high-quality data. The IGMI's databases and maps are structured according to official coding standards. It produces topographic datasets in accordance with both official technical regulations issued by an Italian ministerial decree of 10/11/2011, and NATO international technical specifications for military purposes.

Immediate, up-to-date and suitable data, swiftly supplied, is needed in dealing with an emergency situation. In some cases, a detailed analysis of the main types of objects from a general point of view is required in order to produce an appropriate cartographic representation of the impacted area. These representations are shared with the pertinent civil protection authorities to satisfy post-event activities such as the surveying of damaged buildings or the updating of geographical data.

The IGMI is in its programming activities able to satisfy the objectives described above. They are described in the three phases listed below:

a) A normal, or pre-event, situation; the actions to be undertaken regarding:

- The production of a cartograhic series entitled 50_OPEN. This new series is designed to represent the nation's entire territory in a limited period of time (in few years), compatibly, with updated timing already in adoption in other countries. The content of the vector database is constructed with the principal thematic layers and few basic topological standards; it can automatically consent the production of a related cartographic product at a scale of 1:50000 producing a base from data made available by local administrations and the geographic community. The result is a quicklycreated product originating from a simplified database, but containing the basic information necessary for planning any emergency action regarding transport, urban areas and the hydrological environment.

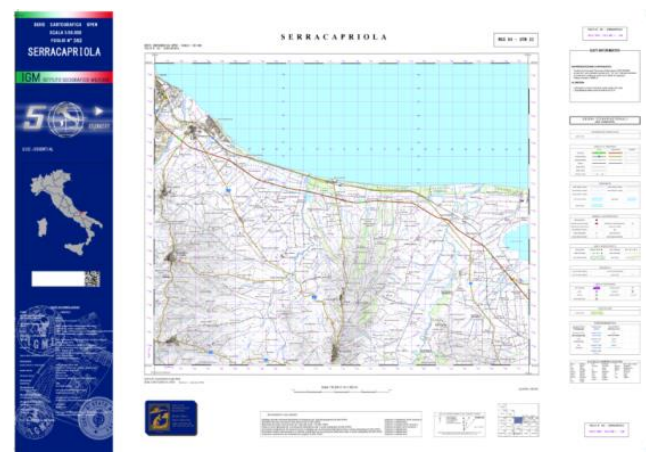

Figure 3. Example of 50_OPEN map

- Having created the 50_OPEN series, the same methodology will be applied to the 1:25000 scale (the new 25_OPEN series) in order to provide more detailed and more accurate information regarding the placement of objects (this will be the future of the IGMI).

- The implementation of some priority thematic layers at the national level beginning with layers regarding hydrology, viability and land use.

- The realization of a national Digital Surface Model (DSM), in conjunction with AGEA to be completed in ca. 3 years, which takes into account the elevation of objects on the ground and having a resolution of 5 meters. This product is is based on the results of remote sensing and land measurments. It allows the extraction of morphological parameters of the surface (for example, gradient/acclimatization, level curves, shaded soil patterns) the orthorectification of aerial or satellite images (AGEA orthophotos of a scale of 1:10000), the acquisition of 3-D rendering, the modelling of mass or fluid movements (landslides, floods, etc.), and the ability to produce for mapping purposes precise geographic and geomorphic information ( Figure 4.).

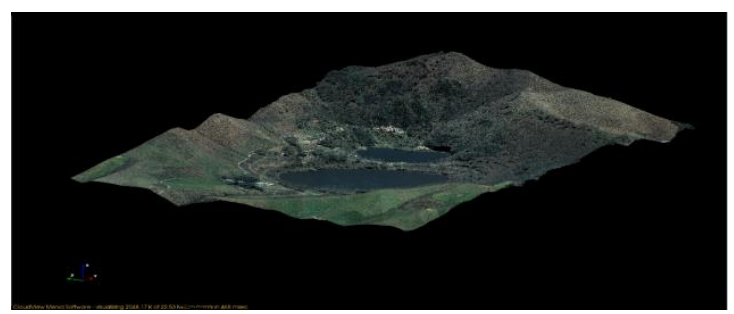

Figure 4. Example of points clouds performed by aerophotogrammetric method with 1 meter resolution

- In addition to quickly- produced products, the IGMI responds to prevention and prediction needs by means of its detailed topographic database, derived principally from regionally supplied data, having a complete contents of a qualitatively superior level. Toward this end, the National Synthesis Database project (Data base di Sintesi Nazionale-DBSN) has been launched in recent years, based on regional data and the integration of missing data from other certified sources or from direct acquisition using the AGEA's orthophotos. The aim of the project is to create a national database through the homogenization of data coming from the Regions and a generalization of the elements contained in order to obtain a level typical of medium scale detail. - The creation of a "crisis map team" to address future emergency situations and able to operate in the field. Toward this end, and in collaboration with the University of Florence Department of Civil and Environmental Engineering (DICEA), specific training is being made available in the form of a Master in "Production and processing of 3D data from groundlevel and drone".

b) An emergency situation; The actions to be undertaken regarding:

- The availability of all geospatial data and cartography produced by the IGMI.

- Intervention in the field of the "crisis map team", land surveying and measures to be taken for management of the crisis.

- Technical support for the upgrading of some types of objects, at a detailed level, such as edifices subject to damage classification to determine a correct reconstruction.

c) Reconstruction phase (post-event); The actions to be undertaken regarding:

- The availability of all geospatial data and cartography pruduced by the IGMI.

- Monitoring activities and geodetic surveying. 
- Technical support in reconstrution activities.

Specifically speaking, the activities that the IGMI can put into operation for the safeguarding of the nation's cultural patrimony follow in this by no means exhaustive list:

- support in the realization of of a georeferenced database of the entire nation's Cultural Heritage;

realization of specific risk maps for the Cultural Heritage; providing support (in the immediate post-event) through its own "crisis map team", in collaboration with trained personnel from other entities, for the monitoring and surveying of cultural assets and the resulting processing of special three-dimensional models;

the organization of specific training activities in the field of geomatics.

\section{7.}

\section{CONCLUSIONS}

The emergency that follows a calamitous event requires proper and updated instruments teritorially informed for a more optimal outcome with respect to those formerly used. It concerns the serious problem of the "quality" of geographical information in general which is particularly present in the "at risk zones", where to have updated data on the built areas, viability, and, in general, on the "infrastructure system", is fundamental for the correct and prompt planning of civil protection intervention, in both the preventive and rescue phases.

Toward this purpose, there should be an availability of uniform geospatial data, updated and shared among all protagonists involved in crisis management. Therefore, it is essential, to begin immediately the gathering of ideas on how to acquire, manage and disseminate widely geospatial data. It is an extremely complex operation which requires precisely co-ordinated planning from the very beginning, first of all at a normative level and only then at technical level.

It is commonly stated that for these purposes it is certainly preferable to have updated documentation resulting from economic and rapid-production criteria (although to some extent at the expense of metric precision) rather than a high quality topographical database but not updated for decades. But I would like to say: "Why is it not possible to have both?". What is preventing the acquistion of high quality geospatial data also adequately updated? All the necessary potentialities are in place, and I do not think that the "economic" factor (let's reflect, a moment, on the major costs of emergency interventions due to inadequate, or, worse still, erroneous geospatial information) that prevent the implementation of a national-level project which can substitute the phrase "extraordinary intervention" with "ordinary intervention", wherein all organizations and institutions dealing with, for whatever reason, the acquistion and production of geospatial information, collaborate with each other. The danger of the lack of comunication and collaboration is the production of an enormous quantity of information which remains, at best, locked inside databases incapable of data sharing.

Another danger to be avoided is the lack of data certification. In an emergency situation anything is possible: "little" becomes better that "nothing". However, in certain cases, this can produce damage perhaps worse than that of the lack of data.

For this reason three phases need to be defined: in the first phase (pre-event) one must plan for the acquisition and production of quality homogeneous geospatial information in a realistic and co-ordinated project, with a duration of at least ten years, involving all the National Mapping and
Cadastral Agencies: (IGMI could play a role of coordinator), the Regions, autonomous provinces, the AGEA, etc. in the production of a database and related cartography at different scales covering the entire national territory. It is obvious that there will be the need for the choices of the priority on information layers to be produced and these must be made based on real necessity of territorial intervention. In this context, and with the same view, the production plan of the Istituto Geografico Militare has started carrying out the geoinformation layers regarding hydrography and viability to which will follow, in chronological order, the builted-areas and the soil-use layers.

Contemporaneously the realization of a project (in collaboration with AGEA) of a 3-level Digital Surface Model (DSM) will enable the creation of orthophotos with the quality necessary for the management of emergency situations while the National Syntesis Data Base (DBSN) project follows the logic of homogenizing and synthesizing at national level the DBs produced at the regional level. In addition, a special mention must be made of "50 OPEN", a new cartographic series possessing a much more rapid production rhythm (7-10 days per sheet) compared to the production capacity of the current series mapping 1:50000 thus, undoubtibly meeting the needs of civil protection.

In the second phase (during the event) it is necessary to intervene immediately in the field supporting civil protection activities by providing all instruments and information necessary for the managment of the crisis, establishing priorities dictated by the situation at hand. The top priority is the saving of human lives, follwed by the safeguarding of the productive and social fabric of the affected areas, in all its aspects, as well as the preservation of historical and Cultural Heritage.

In the third phase (post-event) the availability of updated and certified DBs, as previously described, will ensure better management of the activities related to the reconstruction and the return to normality.

The relative availability of time, compared to the previous phase, however, must not mislead. Time is never enough for the realization of suitable and high-quality mapping tools if the starting point was not adequately prepared during the two previous phases.

It is a well known fact that the Italian topographic databases are insufficient in and of themselves, just as it is equally difficult to take into account the complex issues, which few persons, mainly specialists, are able to evaluate how many and which factors, often in contrast with each other, to use in an intervention seeking to reach a practical solution with its motivations.

The realization of reconstructive interventions renders indispensible the use of methods and the most advanced production techniques which allow for easier updating, in the wait for a return to the"normal" production stage, highlighted in the "pre-event" phase using databases focused first of all on civil protection's needs.

Within this general framework, what are the possible actions relating to the safeguarding of the Cultural Heritage? The 2016 earthquake in central Italy and the consequent loss of Cultural Heritage in the affected territory made even more evident and urgent the need to operate in a co-ordinated manner in the three phases previously mentioned.

The voluntary actions during the event of those persons working assiduously at creating 3-D models to be used profitably during the reconstruction phase has been warmly welcomed and well accepted.

But why not prevent rather that wait for a catastrophic event to occur? Why not to create during the first phase (as it were, 
the "normal"phase) a database structured of 3-D models of the existing Cultural Heritage filled out with a plan, ambitious and long-range, but surely not impossible to achieve?.

This is not an idea recently thought of: even before the catastrophic event of August, 2016 in an invited report to the $61^{\text {st }}$ SIFET conference (the Italian Society of Photogrammetry and Topography conference) the keynote speaker, Professor Grazia Tucci, stated that :

"With regard to the significance of existing built Heritage, our activities (i.e., pertinent University departments) of documentation are part of a preliminary phase, currently supporting actions that will take place in the future. The discipline of restoration has already acquired the concept of "preventive and planned conservation"; so also for us the time has arrived to postulate the establishment of a "preventive digital memory".

It is perhaps superfluous to repeat how important the archives that we produce today are in the event of loss of our patrimony. These same archives of data become precious for the purposes of "virtual restoration" in their simulation of the results of proposed interventions or hypothetical reconstructions of previously existing configurations." (Tucci, 2016).

It is already possible to begin rendering available and sharable currently existing models. I was imagining how many models created by participants in the GeoRes2017 conference could be already included in the database. It would be an starting excellent point.

Another initiative to put immediately in place is the creation of a "geomatic task force" for emergency intervention in crisis areas. This task force made up of trained technical staff (the IGMI, the University, Cadastre, volunteers ...), operationally and logistically independent, would be able to intervene in emergency situations for the surveying of Built Cultural Heritage at risk and with the consequent creation of 3-D models to be used in the third stage of the emergency (reconstruction phase) providing the necessary level of quality of the survey, that, often during an emergency, fail, both from the necessarity and urgency regarding the data availability, and the inadequate experience of the on-site operators.

The "Geomatic Task Force" could be integrated (for now only a hypothesis) by a "crisis map team" formed at the IGM, ready to be deployed immediately when needed, and able to operate in safety and with adequate legal cover, within the organizational structures of the Civil Protection Agency.

For the present, these are simply ideas, possible solutions, requiring, however a serious reflection and consideration. We should not wait for an emergency situation, with the usual helpless attitude in the face of inevitable events to address "institutionally" the problem of the acquisition, production and sharing of geospatial data, "cartographic" products, georeferenced databases, and high quality and homogeneous information layers, regularly updated throughout the national territory. In this context, the IGMI, in its role as National Mapping Agency, is ready to do its part for the protection and safeguarding of the national territory in general and for its Cultural Heritage in particular.

\section{REFERENCES}

Cardillo P.F., Grandoni, D., Di Federico, A.L., 2016. Il support satellitare dell'Euroma per le emergenze, in Geomedia, XX (5), Roma, Italy, pp. 44-46.
Carlucci, R., Cristini B., Fasolo M., 2016. Cartografia per le emergenze, in Geomedia, XX (5), Roma, Italy, pp. 6-14.

Ciasullo, G., Rotundo, A., 2016. Un possibile ruolo del RNDT per l'accesso e l'utilizzo dei dati territoriali nella gestione delle emergenze, in Geomedia, XX (5), Roma, Italy, pp. 54-56.

Conte, A., Bonofoglio, M.P., Petriglia, L., 2016. Il Geoportale Nazionale: un'infrastruttura a support delle emergenze, in Geomedia, XX (5), Roma, Italy, pp. 58-60.

Ferrante, F.C., Ambrosanio M., M., 2016. Il sistema cartografico del catasto quale strumento di supporto alla gestione delle emergenze, in Geomedia, XX (5), Roma, Italy, pp. 36-39.

Surace, L., 2012. Data base Topografici e calamità naturali, in Atti $16^{\wedge}$ Conferenza Nazionale ASITA, 6-9 novembre 2012, Vicenza, Italy, pp. 1-4.

Tucci, G., 2016. Geomatics and digital heritage. From dataset to models: what next?, in Bollettino SIFET, Sezione Scienza, 2016 (3), Cagliari, Italy, pp. 1-10. 